\title{
L'animation d'un espace numérique de discussion : l'exemple des forums usenet
}

\author{
Michel Marcoccia \\ Laboratoire Tech-CICO/Université de Technologie de Troyes \\ 12, rue Marie Curie, BP2060 \\ F-10010 Troyes cedex \\ michel.marcoccia@utt.fr
}

\begin{abstract}
RÉSUMÉ. Cet article est consacré à l'émergence du rôle d'animateur dans un forum de discussion usenet non modéré. Nous définirons dans un premier temps les spécificités d'un forum de discussion en tant que document numérique. Puis, à partir de l'analyse conversationnelle d'un forum de discussion francophone non modéré, nous décrirons la manière dont un participant de forum peut occuper le rôle d'animateur «occasionnel » en adoptant un comportement interactionnel adapté. Nous verrons alors que ce rôle permet d'assurer la cohérence de l'espace numérique de discussion et aussi d'occuper le leadership dans cet espace, car il repose sur un certains nombre d'actes de langage qui sont des marqueurs de position haute dans la conversation.

ABSTRACT. This paper deals with the emergence of the role of host in a non-moderated usenet newsgroup. First, we will define the specific features of a newsgroup as a digital document. Secondly, through the conversational analysis of a French-speaking non-moderated newsgroup, we will describe the way an user of a newsgroup can play the role of "occasional host", adopting a particular interactional behaviour. At last, this paper will show that playing the role of host permits the coherence of the digital space of discussion and is a way to take the leadership in this space, because speech acts relative to this role are also markers of high position in conversation.

MOTS-CLÉS : animateur, forum de discussion, document numérique, analyse conversationnelle. KEYWORDS: host, newsgroup, digital document, conversational analysis.
\end{abstract}

Document numérique. Volume $5-\mathrm{n}^{\circ} 3-4 / 2001$, pages 11 à 26 


\section{Introduction}

Les forums de discussion sont des espaces numériques de discussion qui permettent à des utilisateurs de gérer des activités intellectuelles collectives, que ce soient des simples discussions ou des processus complexes de résolution de problèmes ou d'aide à la décision. Les forums de discussion sur l'internet (de type usenet) offrent à celui qui les analyse la possibilité d'observer de nombreux phénomènes intéressants, autant du point de vue des formes de cognition collective que des possibilités de gestion coopérative par les acteurs de leurs activités en communauté.

Cet article ${ }^{1}$ sera consacré à un phénomène particulier : l'animation d'un forum de discussion non modéré. En utilisant les méthodes de l'analyse conversationnelle, basées sur l'empirisme descriptif et fondées sur les bases épistémologiques de l'ethnométhodologie ou de l'interactionnisme symbolique (de Luze, 1997, de Queiroz \& Ziolkovski, 1994), nous décrirons la manière dont un forum de discussion non modéré se constitue en «communauté de parole en ligne » (Marcoccia, 2001a, p. 182-183) produisant un document numérique « collectif ».

Dans un premier temps, nous mettrons en évidence les caractéristiques qu'ont les forums de discussion en tant que documents numériques. Partant des définitions de la littérature sur la question, on verra que les forums de discussion peuvent être caractérisés comme des documents numériques dynamiques, collectifs et interactifs. Nous verrons alors que l'animation de l'espace de discussion numérique que constitue le forum est une activité centrale pour l'établissement même du forum comme document.

Ensuite, nous nous attacherons à décrire le type de comportement discursif et communicatif permettant à un utilisateur du forum d'occuper un rôle d'animateur occasionnel. Enfin, nous mettrons en relation ce rôle d'animateur avec la question des relations hiérarchiques à l'intérieur du forum. Partant du modèle des systèmes de place (Kerbrat-Orecchioni, 1992, chap.2), nous verrons que le type d'activités conversationnelles pris en charge par l'animateur lui permet, du même coup, d'occuper une position haute dans l'interaction.

Ce travail est basé sur l'analyse conversationnelle de cent messages adressés au forum de discussion francophone fr.rec.boissons.vins (50 messages en juin et juillet 1997, et 50 messages en avril 1999). Ce forum de discussion usenet est un forum thématique permettant à des amateurs de vins d'échanger des conseils, de poser des questions, de s'échanger des adresses, sans jamais entrer véritablement dans un discours technique sur la viticulture.

1. Cet article est une version développée d'un travail de recherche initialement présenté dans le cadre du colloque «Coopération, innovation et technologies », organisé par l'Université de technologie de Troyes, en novembre 2001. Cf. Marcoccia, 2001b. 


\section{Les forums de discussion : des documents numériques dynamiques}

On peut à première vue considérer un forum de discussion comme un document numérique constitué d'un regroupement d'articles consacrés à un même thème et postés par les abonnés du forum. En tant que produit d'une activité de documentation, un forum est indiscutablement un document numérique. Mais si l'on s'intéresse à l'activité qui a conduit à ce produit, on observe que les forums de discussion sont en fait des documents numériques problématiques, pour lesquels la question de la gestion et de l'animation est centrale.

En effet, on considère généralement qu'un document (numérique ou non) rassemble de l'information en un objet unique et fini. Si l'on s'en tient au critère du support et du produit final, les forums de discussion usenet sont clairement des documents numériques. Mais, lorsqu'on s'intéresse à leurs procédures de génération, les documents numériques en général, et les forums en particulier, touchent au limite des définitions de base du document.

Un premier point est signalé par Stern (1997) : certains documents numériques sont mis à jour en temps réel et ne sont donc jamais véritablement finis. Peut-on encore parler de documents ou ne doit-pas parler d'espaces (ou d'interfaces) d'information ou de communication dynamique ? On peut noter qu'une question tout à fait similaire se pose lorsqu'on tente de définir un forum de discussion comme objet d'étude d'une analyse de discours. Classiquement, l'objet de l'analyse des discours est une archive, qui regroupe un ensemble de textes référés à un même positionnement, ce qui garantit la cohérence et la pertinence de l'objet d'étude (Maingueneau, 1991, p. 22). Les critères de clôture de l'archive deviennent alors déterminants pour la qualité de la démarche de l'analyste de discours et de ses résultats. Un forum de discussion est alors un objet hybride pour l'analyse de discours, à la fois une archive identifiable par un support et un contenu et une conversation qui n'est jamais totalement finie. Il faut alors considérer les forums comme des documents numériques dynamiques, des documents comme objets de processus, ou, pour prendre les termes de l'analyse de discours, comme une archive en train de se constituer (en l'occurrence, une «archive conversationnelle »). On peut donc dire qu'un forum de discussion constitue un document car il produit une «conversation persistante» (Erickson, 1999), combinant la dynamique de la conversation en face à face avec la stabilité de l'écrit. Ce type de document repose sur une structuration hiérarchique de documents numériques à trois niveaux différents: un message posté dans un forum, un sujet composé de plusieurs messages, un forum composé de différents sujets ${ }^{2}$.

Un autre point est évoqué par Stern (1997) : un document numérique peut ne pas être sous la responsabilité d'un éditeur qui en garantirait l'unicité mais peut être

2. On peut noter que message, sujet et forum appartiennent tous les trois à la typologie des genres de documents numériques proposée par l'Université Laval (Québec).

Voir www.bibl.ulaval.ca/doelec/doelec29.html 
composé de données issues de différentes sources. On peut alors soit considérer qu'il s'agit de documents composites (des documents de documents), soit qu'ils sont produits par un processus d'écriture collective. La participation à un forum peut ainsi être vue comme la participation à l'écriture collective d'un document, ou d'un projet (Lewkowicz \& Zacklad, 1999). Un forum de discussion peut donc être défini comme un document numérique dynamique et collectif.

Par ailleurs, dans sa définition la plus simple, un document est une trace de l'activité intellectuelle humaine, conçu dans l'objectif d'être interprété (lu, vu, écouté) par des personnes différentes de ceux qui l'ont mis en place. Un autre problème apparaît lorsqu'on veut caractériser les forums de discussion comme des documents numériques. En effet, la distinction entre l'instance de production du document et son instance de réception n'est plus pertinente. Ainsi, un forum de discussion instaure un cadre participatif complexe (Marcoccia, 2002) beaucoup plus proche de celui de la conversation que de celui de la relation éditeur-lecteur. Tout participant peut à sa guise osciller entre différentes formes d'engagement : récepteur des informations contenues dans le document (lecteur), producteur occasionnel ou régulier d'informations. Le format de réception des messages contenus dans un forum est aussi complexe, du simple lecteur au destinataire «adressé », en passant par les destinataires indirects ou privilégiés. Un forum de discussion est de ce point de vue un document interactif (ou interactionnel) au sens strict, c'est-à-dire ne reposant pas sur l'opposition émetteur-récepteur. On verra alors que le rôle d'animateur est nécessaire pour que l'addition des contributions au forum constituent un document cohérent (et non plus seulement un document composite), dans la mesure où l'animateur vient en quelque sorte occuper le rôle d'éditeur, qu'il soit préalable ou ratifié de manière collective et coopérative par les autres participants.

Le forum de discussion est un espace numérique qui pose aussi la question de la cohérence du document que constitue l'ensemble de ces messages. On considère qu'un document est une unité autosuffisante représentant une contribution intellectuelle, publié sur un média pour des raisons spécifiques. Un document exhibe ainsi une structure intentionnelle qui définit comment les éléments de son contenu sont organisés selon des axes dans l'objectif d'être interprétés par un lecteur comme témoignage de cet objectif original de publication (Auffret \& Bachimont, 1999). Selon Bachimont (1999), un certain nombre de contraintes découlent du support d'inscription et de la forme sémiotique choisie, déterminant l'expression du contenu et ses conditions de réception et d'interprétation. En particulier, deux types de contraintes émergent :

- une structure logique qui permet la cohérence et l'ordonnancement des éléments de contenus exprimés selon un mode d'expression donné ;

- une structure matérielle imposant une présentation de ces éléments.

Les différentes structures internes au document sont mises en place dans un contexte de production, dans le cadre d'une pratique donnée caractérisant un genre 
du document qui va déterminer les contraintes de production du document et aussi son contexte de réception.

Le rôle de l'animateur d'un forum de discussion sera en quelque sorte celui d'un documentaliste, chargé d'assurer la cohérence des messages contenus dans le forum, aussi bien du point de vue de leur contenu (focalisation thématique), que de leur structure (normes de rédaction ou taille des messages par exemple). Ainsi, les spécificités des forums de discussion comme documents numériques sont multiples. Ce sont des documents dynamiques, produits collectivement de manière interactive, et dont la cohérence du contenu et de la forme ne peut être que le résultat d'une gestion collective et coopérative. Les participants à un forum de discussion ont une sorte de responsabilité partagée dans la production d'un document cohérent, dont la réception et l'appropriation par un utilisateur est facilitée. L'animateur d'un forum joue un rôle central pour la gestion de ce document numérique particulier.

\section{L'animation d'un forum de discussion}

\subsection{Définitions}

La manière dont les discussions vont se dérouler dans un forum diffèrent grandement selon que le forum est modéré ou non. Au sens strict, lorsqu'un forum est modéré, les messages qui sont postés sont en fait adressés au modérateur qui choisit de les diffuser dans le forum ou de les exclure de la discussion. Dans ce cas, la modération du forum correspond clairement à une activité d'édition d'un document numérique dont la représentation affichée sur le support d'appropriation sera l'œuvre d'un éditeur particulier garantissant la cohérence du document par la sélection voire le «reformatage » des messages adressés au forum. Les critères de sélection sont souvent assez hétérogènes; ils dépendent tout autant de la charte du forum (par exemple pour la cohérence du contenu) que de la nétiquette en général (Marcoccia, 1998). Ils sont aussi très largement déterminés par la représentation que le modérateur se fait de sa fonction (Berge \& Collins, 2000).

Quelques travaux ont déjà été consacrés au rôle de modérateur, particulièrement dans des études en CSCL (Computer-Supported Cooperative Learning). Les résultats principaux de ces travaux montrent que le rôle de modérateur se caractérise par une configuration de divers rôles : facilitateur (le modérateur est un médiateur), «manager » (administrer, archiver, gérer les inscriptions), filtre (accepter/rejeter des messages), expert (gérer les Foires-Aux-Questions), éditeur (éditer les textes, assurer leur correction, assurer la production du document numérique final), animateur de la discussion (poser des questions, garder la discussion «dans le fil »), «marchand » (marketer, promouvoir le document), secours (helper), «pompier»(firefighter, rejeter les messages injurieux, protester) (Berge \& Collins, 2000, p. 90). L'analyse de certains rôles a été développée : par exemple le rôle d'éditeur a été comparé au rôle d'éditeur dans le champ journalistique, du point de vue des implications 
techniques, déontologiques ou juridiques (Morris, 1993). D'autres travaux ont été consacrés aux motivations des modérateurs, aux raisons justifiant la modération d'un forum ou au partage des rôles (par exemple Berge \& Collins, 1997).

Ces travaux apportent des résultats intéressants pour l'analyse du rôle d'animateur. Néanmoins, ces résultats ne permettent pas de comprendre des mécanismes de gestion coopérative et collective de forums, marquée par l'émergence du rôle d'animateur et non pas par l'identification préalable d'un éditeur. Ainsi, on voit bien que le rôle d'animateur n'est qu'une partie du rôle de modérateur et ne se confond pas strictement avec celui d'un éditeur de document produit par une écriture collective. Il convient de noter que les travaux sur les forums modérés ne parlent d'ailleurs que de «modérateur» (e-moderator) alors qu'on réserve le terme d'animateur (host) lorsqu'on traite de forums non modérés. Le rôle de modérateur nous semble en fait beaucoup plus lié à celui d'un éditeur, dont les tâches principales peuvent se résoudre par la simple procédure de sélection des messages. Ce sont seulement les modalités de participation au forum qui font du modérateur un animateur. D'ailleurs, la participation à l'interaction n'est pas une condition nécessaire de la modération d'un forum, qui se peut limiter au travail d'édition a posteriori d'un document résultant d'échanges préalables de messages. Enfin, le fait que le modérateur occupe un rôle préalable met au premier plan les critères organisationnels ou sociaux qui ont présidé à sa nomination (qu'elle soit autoproclamée ou non). Le rôle de modérateur est un rôle institutionnel dont la part interactionnelle (la manière dont le modérateur se comporte) pourra n'être que la conséquence de l'institution.

Les messages qui constituent notre corpus sont extraits d'un forum non modéré. Du point de vue de la dynamique des groupes, on peut qualifier ce type de forum de «groupe informel », c'est-à-dire de groupe dont les règles de fonctionnement émergent progressivement des interactions (Maisonneuve, 1986, p. 18). Dans ce cas, l'animateur est un rôle occasionnel ${ }^{3}$. Cela signifie que c'est un rôle uniquement interactionnel, qui n'est pris en charge que par l'adoption d'un comportement interactionnel qui sera reconnu par les autres interactants comme permettant d'occuper un rôle particulier, dans le cadre d'une gestion collective et coopérative de l'espace de discussion. De ce point de vue, on dira que le rôle d'animateur correspond à un type d'activités caractérisant une identité située. Peu de travaux ont été consacrés au rôle d'animateur comme identité située ou comme rôle occasionnel. Beaudoin et Velkovska (1999) ont mis au jour le type d'activités propres à certains rôles dans les forums (par exemple les rôles d'habitué et de nouveau) et ont abordé la question de la validation du leader dans un forum, réduit plus ou moins au rôle d'expert (Beaudoin \& Velkovska, 1999, p. 165).

Si l'on trouve peu de travaux sur le rôle occasionnel d'animateur, on trouve en revanche de nombreux textes prescriptifs, sur l'art d'animer une discussion en ligne. Ainsi, Howard Rheingold (qui est un des promoteurs essentiel de la communauté 
virtuelle comme activité en ligne) définit le rôle de l'animateur en listant les objectifs suivants (Reinghold, 1998) : susciter des conversations « authentiques », faire naître un sentiment d'appartenance au groupe, générer de la créativité collective, favoriser l'autogestion des conflits, permettre les conditions de la collaboration entre membres du groupe, lutter contre la passivité des participants, accueillir les nouveaux arrivants. On retrouve essentiellement l'aspect socio-affectif de la fonction de leadership, telle qu'elle est habituellement décrite en dynamique des groupes (Maisonneuve, 1968), comme si le rôle d'animateur était uniquement un facteur de cohésion du groupe. Rheingold propose par ailleurs un certain nombre d'analogies pour définir le rôle d'animateur : l'animateur est comme un « hôte », l'organisateur d'une soirée. Il est aussi une autorité, voire un exemple pour les autres membres du groupe. Il est un «cybliothécaire» (cybrarian, un éditeur électronique). On reprendra la question de l'autorité pour montrer l'importance du rôle d'animateur dans la gestion des conflits. Ce type de mode d'emploi du rôle d'animateur nous semble définir plus le profil psychologique de l'animateur que le type d'activités liées à ce rôle. Ainsi, lorsqu'il décrit le Host Behavior, Rheingold insiste sur la civilité, la patience, la précaution, l'humour, l'élégance et autres qualités morales nécessaires à l'animateur. Lorsque des activités sont mentionnées, elles le sont de manière très générale.

\subsection{Les comportements communicationnels de l'animateur}

Dans cet article, nous allons proposer une description des tâches effectuées par le participant qui joue le rôle d'animateur, en montrant que ces comportements discursifs ou interactionnels répondent à trois objectifs fondamentaux.

De manière empirique, nous avons observé pendant trois ans (de 1997 à 1999) le forum de discussion fr.rec.boissons.vins, sans prendre part aux discussions. Nous avons régulièrement enregistré des échantillons de 50 ou 100 messages (pour cet article, le corpus est composé du plus ancien et du plus récent échantillon, chacun composé de 50 messages). En étudiant ces messages, on a pu constater la prise en charge progressive du rôle d'animateur par un participant particulier (nous l'appellerons Paul) et de sa transformation en «leader» du forum (nous entendons ici «leader» dans son sens français, si l'on peut se permettre cette terminologie paradoxale).

Qui est ce participant? Adoptant une perspective ethnométhodologique, nous n'avons pas cherché à avoir plus d'information sur Paul que ce qu'en ont les autres participants. La signature de ses messages indique son appartenance professionnelle (Paul envoie ses messages à partir de son ordinateur professionnel). Rien dans cette appartenance ne permet ni de le distinguer des autres participants, ni d'établir son niveau d'expertise dans le domaine du vin: Paul est employé dans une entreprise n'ayant aucun rapport avec la viticulture. Sa capacité à occuper le rôle d'animateur est donc uniquement liée à son profil communicationnel distinctif, qui constitue un 
ensemble de comportements dont la corrélation nous semble définir le rôle d'animateur. Quels sont ces comportements ?

\subsubsection{Occuper le terrain}

Paul est le participant qui envoie le plus de messages (18\% du corpus total). Ce critère, peu évoqué dans la littérature descriptive ou prescriptive, ramène à une composante simple de l'interaction : « occuper le terrain ». Par ailleurs, on peut considérer que cette activité contribue indirectement à la cohérence du document numérique constitué par la somme des messages adressés au forum.

\subsubsection{Diriger et organiser la discussion}

L'animateur occasionnel dirige la discussion. Ce comportement attendu se manifeste par les activités suivantes: il initie de nouvelles séquences, il pose de nouvelles questions et crée de nouveaux fils du discours, comme dans l'exemple $1^{4}$.

\section{Exemple 1:}

J'ose ouvrir, et ce n'est pas sans apprehension, un debat sur les associations de vins avec les fromages. Je l'avoue, je suis constamment inquiet quand arrive le moment de mettre le plateau de fromages sur la table.

Je suis convaincu qu'il existe des associations " presque » inevitables comme le Sancerre blanc ou le Menetou-Salon blanc avec un morceau de chevre blanc; ou alors un vin jaune avec le comte (surtout si on rajoute des noix); ou un liquoreux avec un bout de roquefort.

Cela dit, lors d'un repas, il n'est pas toujours facile de realiser tous ces accords. En effet, certains ne concoivent pas deboucher un vin blanc sec apres le Saint-Estephe ouvert pour le sanglier. Faut-il, de toutes manieres, deboucher une bouteille de Sauternes sur la fourme d'Ambert alors que la bouteille de Minervois est a peine entamee?

D'autres questions me viennent a l'esprit? Quel type de pain faut-il pour faire ressortir le gout du fromage, en association avec le vin: du pain grille, du pain blanc, aux cereales? Enfin, y-a-t'il des regles de base concernant tel ou tel type de fromage (pates pressees cuites/non cuites, etc.)

Quelles sont vos experiences personnelles?

Cordialement.

[signature]

L'animateur peut aussi proposer la clôture d'un fil discursif, comme dans l'exemple 2.

Exemple 2 : (extrait d'un long message) : Paul répond à différents messages sur la qualité des vins faits avec du gamay, il donne son opinion et conclut :

En conclusion, je dirais que, meme si tous les gouts sont dans la nature, il existe de tres bons vins a base de gamay.

4. Les exemples cités sont reproduits tels quels. Seuls les noms des participants ont été modifiés. 
La régulation de la conversation peut amener l'animateur à demander à un participant de reformuler son message, comme dans l'exemple 3.

Exemple 3 :

John Smith wrote :

$>$

$>$ I would highly recommend that you all grab a bottle of the 1995 Domaine > Clavel

"La Copa Santos" of Provence. I picked up a bottle for $\$ 17.00$ US > at a wine store near Columbus Circle.

> It carries a big 94 rating from Robert Parker, Jr., and it's the best under

$>\$ 20$ bottle of wine I've > had in a couple of years.

> Truly profound.

C'est surement tres bon, si vous le dites. Je souhaiterais simplement que vous nous en parliez mieux.

Cordialement.

Dans ce message multilingue, l'animateur joue à la fois un rôle de facilitateur (il aide Scott dans son travail de formulation), d'éditeur (il contrôle la forme des messages), et, du coup, joue a posteriori le rôle de filtre que joue a priori un modérateur. On voit bien l'importance qu'ont ces trois activités (facilitateur, éditeur, filtre) pour l'élaboration dynamique et collective d'un document numérique. En effet, ces activités peuvent permettre de garantir la cohérence du contenu et de la forme des messages constituant au fil du temps un document numérique qui peut offrir un contexte de réception satisfaisant. En d'autres termes, l'animateur permet plus ou moins de garder le forum dans un cadre générique (les règles d'un genre) facilitant sa réception.

\subsubsection{Modérer la conversation}

L'animateur s'attache aussi à modérer la conversation en rappelant les règles de conduite acceptables dans un forum de discussion. Le contrôle des conduites est une tâche centrale dans les forums de discussion (Smith, McLaughlin \& Osborne, 1997), ce dont témoigne le nombre très important de commentaires métadiscursifs observables dans les forums: de très nombreux messages sont consacrés au commentaire et à l'évaluation de la manière dont d'autres messages ont été rédigés (Marcoccia 2001c). En fait, sans participant rappelant les règles de « savoir-vivre », l'anonymat des participants et l'absence de coprésence physique risquerait de générer des discussions agressives et du coup de parasiter la production collective du document. Deux types de règles de conduites sont rappelées :

a) les règles qui doivent être respectées dans le cyberespace en général. Ce sont les règles de la nétiquette, qui conseillent de résister à la tentation d'incendier (flame) autrui, d'être trop agressif ou ironique, par exemple. C'est ce type de règle qui est invoquée dans le message 4 : 


\section{Exemple 4 :}

Zorba wrote :

$>$

> Le muscadet, c'est pas du vin, c'est de la piquette. N'est-ce pas les nantais?

C'est *typiquement* le genre de "thread" qui n'a pas sa place ici.

b) les règles de conduite spécifiques au groupe de discussion. En général, les groupes de discussion, dès lors qu'ils se constituent en communauté de parole (Marcoccia, 2001a), tendent à développer des normes comportementales particulières, relatives à l'objectif ou à l'idéologie du groupe. Dans le forum sur le vin, les messages à caractère commercial sont interdits. Cette règle est rappelée par l'animateur dans l'exemple 5 :

\section{Exemple 5 :}

Tim a ecrit :

$>$ surement les meilleurs prix du marché

$>$ http ://www.vins-fr.com

Puis, il a ajoute :

$>$ je suis desole de ne pouvoir satisfaire tout le monde.

> N'hesitez pas a communiquer les sites qui offrent mieux.

Surement pas!

Ce forum n'est la pour parler de tarifs, mais pour parler de vins. C'est clair, non?

Ces trois types d'activités constituent le profil communicationnel de base d'un animateur. Cela signifie qu'un participant peut occuper le rôle d'animateur en adoptant ce profil, même si rien dans son identité ne le distingue des autres participants. C'est le cas dans ce forum, où aucun autre participant ne vient remettre en cause l'animateur occasionnel. On tentera d'expliquer ce phénomène plus loin. En fait, l'animateur n'est pas mis en concurrence avec d'autres participants prétendant jouer son rôle. Au contraire, un participant le seconde, en renforçant de manière coopérative les activités de l'animateur (par exemple en réitérant un rappel à l'ordre) ou en rappelant sous une forme explicite les règles que l'animateur a invoquées implicitement. Concrètement, ce coanimateur s'est chargé de rédiger une charte, ce qui est une pratique assez courante dans un forum, mais cette charte est apparue dans le forum bien après la prise en charge du rôle d'animateur par Paul, comme si elle venait expliciter et renforcer ce rôle.

Certains traits du rôle d'animateur correspondent à sa prescription. Cela signifie, pour ces traits, que le rôle tel qu'il est prescrit est éventuellement connu des participants et qu'il fonctionne alors comme un script à partir duquel on évalue la capacité qu'ont certains participants à se prétendre animateurs. 


\section{Animation et prise de leadership dans l'espace numérique de discussion}

Si l'on s'arrête à ce niveau de description, on observe déjà que le rôle d'animateur est très proche du rôle de «leader ». En termes plus interactionnistes, on dira que le rôle d'animateur permet à celui qui l'occupe de se mettre en position haute dans l'interaction entre les différents participants contribuant à l'écriture collective du document. En effet, «parler» plus que les autres, gérer la discussion (ouvrir et fermer des séquences thématiques), rappeler les règles de conduite sont de manière prototypique des marques de position haute, si l'on admet une théorie taxémique des conversations (Kerbrat-Orecchioni, 1992). Par exemple, le message 3 montre que l'animateur prend la responsabilité de produire un message relativement menaçant pour la face de son destinataire, et donc, se met en position haute.

On doit noter que celui qui joue le rôle d'animateur occupe aussi très largement une position d'expert dans le forum. Cette expertise se manifeste de plusieurs manières. Par exemple, il envoie des messages plus longs que les autres et dont le contenu est souvent plus «technique », comme dans l'exemple 6 :

\section{Exemple 6 : (extrait)}

La surmaturite et le botrytis n'empechent pas (heureusement :-) de vinifier un vin sec. Les Coteaux-du-Loir du domaine de la Belliviere sont aussi vinifies comme cela. Le vin est moelleux quand il y a du sucre residuel.

De plus, l'animateur établit son autorité et son expertise lorsqu'il ratifie les réponses et les opinions des autres participants :

\section{Exemple 7 : (extrait)}

> pourquoi parler de grands vins en oubliant de parler de ce fameux chateau

$>$ chalon.ce vin de predilection ne devrait jamais etre dissocie des grands vins

$>$ francais. (...)

Vous avez raison de dire que ce vin fait partie des plus grands vins francais.

(...)

Enfin, l'animateur est le participant qui est le plus souvent en position d'adresser des messages contenant des conseils, acte de langage qui met celui qui le produit en position haute :

\section{Exemple 8 : (extrait)}

Columbo wrote :

$>$ Bonjour,

> Devant aller passer le mois de mai a Nantes et ne connaissant pas du tout la > region, une ame charitable

> pourrait-elle m'indiquer des noms de vins a decouvrir dans cette region.

He bien, il y en a pas des caisses:-)

Je conseille toutefois, bien qu'il s'agit de grands classiques dans le Muscadet :

- Le Chateau de la Preuille, a St-Hilaire-de-Loulay.

- Le Domaine de l'Ecu, au Landreau (ma preference).

- Le Domaine de la Louvetrie, a la Haye-Fouassiere. 
- Ollivier pere et fils, a Maisdon-sur-Sevre.

- Le Chateau de Chasseloir, a St-Fiacre-sur-Maine.

Pour decouvrir les muscadets, on peut aller faire un tour du cote de la cave de Longchamp, rue Georges-Laffont, a Nantes.

La position haute - ou le leadership - liée au rôle d'animateur est ratifiée par les autres participants. Par exemple, l'émetteur d'un message peut indiquer son accord avec l'animateur, dont il reproduira les énoncés :

Exemple 9 :

> En simple degustation, entre amis, les côteaux du Layon sont superbes. En > accord avec les mets, je le preconise plutot avec des fromages bleus

$>$ (roquefort, fourme, etc), ou en dessert sur des sorbets.

Oui, oui!

Comme pour du Sauterne, en apéritif, très frais, et comme le signale Paul, des toasts au Roquefort iront très bien.

L'exemple 9 est représentatif d'un processus de gestion coopérative de l'espace de discussion; il signale la manière dont les utilisateurs d'un forum peuvent manifester le fait qu'ils acceptent l'autorité d'un des leurs, en confirmant la qualité de ses opinions et de ses conseils. Quelle est alors la relation entre la reconnaissance d'un participant comme animateur et son acceptation comme leader? L'animateur devient-il le leader, ou bien est-ce l'inverse ? On pourrait en effet penser que c'est la reconnaissance de l'expertise d'un participant qui lui permettrait d'occuper le rôle d'animateur. Cette hypothèse, qui est sociologiquement valide, voire banale, est infirmée par nos données. En effet, d'autres participants du forum fr.rec.boissons.vins occupent nettement des rôles d'experts, facilement identifiables par les indices discursifs contenus dans leurs messages. Mais aucun de ces experts ne dispute à l'animateur le droit d'occuper son rôle, qui parait donc être le résultat d'une validation interlocutive et coopérative, à partir de laquelle se construit la confiance collective en la légitimité de l'animateur (Hauch \& Lebraty, 1998).

\section{Animer la communauté virtuelle}

On peut sans doute comprendre les raisons de la ratification collective du droit qu'a un participant de jouer le rôle d'animateur et de prendre en même temps le leadership du groupe, si l'on s'intéresse aux conditions d'émergence et de réussite d'une communauté virtuelle.

A partir de quel moment un groupe d'individus échangeant des messages se constitue-t-il comme communauté en paroles (Parret et al., 1995) ? En s'appuyant sur différents travaux dans le champ des Computer-Mediated Communication Studies (Baym, 1998 ; Erickson, 1997; Kollock \& Smith, 1996 ; DuVal Smith, 1999, par exemple) on peut dégager neuf conditions qui doivent être remplies pour qu'un groupe conversationnel en-ligne se constitue en communauté en paroles (Marcoccia, 2001a) : 
- le sentiment d'appartenance des membres (et corrélativement le sentiment d'exclusion);

- la possibilité pour les membres de construire leurs identités dans la communauté ;

- l'importance de la dimension relationnelle des échanges ;

- l'engagement réciproque des membres ;

- le partage des valeurs et des finalités du groupe ;

- l'émergence d'une histoire commune ;

- la durée des échanges ;

- l'existence de principes de pilotage des comportements des membres du groupe et de mécanismes de résolution de conflit dans le groupe ;

- la réflexivité du groupe.

Quels comportements interactionnels vont permettre à ces conditions de se réaliser, en d'autres termes, quels sont les comportements favorisant la constitution d'un groupe de discussion en ligne en communauté en paroles ? On observe qu'une grande partie de ces conditions peuvent être remplies dès lors qu'un animateur va émerger et adopter les comportements communicationnels adaptés.

Ainsi, le sentiment d'appartenance au groupe pourra se renforcer si les membres du groupe ont un modèle de comportement fonctionnant comme pôle d'identification. C'est sans aucun doute l'un des rôles de l'animateur. De même, ce sentiment d'appartenance sera d'autant plus fort que la définition du groupe sera homogène, ce à quoi contribue aussi le rôle d'animateur (en cadrant les discussions par exemple). La qualité de la dimension relationnelle des échanges sera aussi protégée par les interventions de l'animateur. Le partage des valeurs et des finalités peut être construit ou renforcé par l'explicitation de l'éthique du groupe, tâche prise en charge par l'animateur. L'émergence d'une histoire commune, d'une mémoire du groupe, est aussi largement favorisée par la prise en charge «technique»par l'animateur d'opérations de synthèses, de reformulations, de bilans. Enfin, DuVal Smith (1999) insiste sur un point important : une communauté virtuelle n'existe que si elle se dote de méthodes de gestion des conflits. En effet, une communauté en ligne se distingue des groupes en face à face par l'ouverture de ses frontières, l'anonymat relatif de ses membres et la possibilité d'une grande diversité sociale et culturelle. Chacun de ses aspects peut favoriser le conflit. On peut alors penser que l'émergence d'un animateur et sa ratification par les autres est un moyen de gérer le conflit, très peu « coûteux » pour les autres participants.

En d'autres termes, l'animateur est ratifié collectivement en échange de ses «bons services ». L'acceptation du leadership qui découle de son rôle d'animateur peut être vue comme un salaire symbolique. Comme le montre Donath (1999), l'établissement de la réputation et la reconnaissance par les autres jouent un rôle central dans l'investissement que certains consacrent à animer des communautés en ligne. 


\section{Conclusion}

Cet article montre dans un premier temps la nature particulière d'un forum de discussion en tant que document numérique ; c'est un document dynamique produit par un processus d'écriture collective et interactive. On pourrait parler de document numérique conversationnelle ou de conversation comme archive numérique.

Nous avons ensuite défini le rôle d'animateur en montrant qu'il ne peut pas être confondu avec le rôle de modérateur. Le rôle d'animateur se constitue essentiellement par l'adoption de comportements communicationnels adaptés à un triple objectif : occuper le terrain, organiser la conversation dans sa forme et son contenu, contrôler le respect des règles de conduite. La prise en charge de ce rôle ne donne pas nécessairement lieu à de la compétition, alors même qu'il assure une position haute à celui qui l'occupe. L'importance de l'animateur dans l'émergence, le renforcement et le maintien d'une communauté virtuelle permet sans doute de comprendre la ratification collective de cette identité située. En particulier, déléguer à un animateur l'autorité des membres du groupe sera un moyen efficace de gérer les conflits.

Enfin, ce travail nous a permis de voir en quoi le rôle de l'animateur est tout à fait central dans le processus de génération du document numérique constitué par l'ensemble des contributions au forum. L'animateur intervient dans la forme du document : la taille des contributions, leur «formatage», leur style sont autant de dimensions gérées par l'animateur, ou, au moins gérées de manière coopérative par un collectif animé et dirigé par l'animateur. Le contenu du document, la somme de connaissances consultable et la possibilité de constituer ce document en archive identifiable et consultable dépendront largement de la manière dont l'animateur a réussi à maintenir une focalisation thématique cohérente. Dans le cas d'un forum de discussion, la structure du document correspond en fait à l'organisation hiérarchique des échanges, dont les principes de cohérence et, donc, de lisibilité peuvent être maintenus par l'animateur. Enfin, l'identité même du document dépendra très largement de la manière dont l'animateur aura réussi à organiser et à diriger la gestion coopérative et collective du forum. Ces différentes dimensions du rôle de l'animateur permettent bien de faire d'un espace numérique de discussion un document numérique dynamique et collectif car elles assurent le maintien d'un cadre générique au document, permettant sa consultation et son enrichissement collectif.

Cet article pose en fait les bases d'un travail de recherche qui devra être appliqué à d'autres formes de forums pour que ses résultats soit validés. Ainsi, diverses perspectives de recherche pourront être développées, par exemple en essayant de saisir précisément la manière dont l'animation d'un espace numérique de discussion dans une organisation permet la gestion et la mémoire des archives de cette organisation dans une perspective de gestion des connaissances. Quel rôle joue l'animateur d'un espace numérique de discussion dans la constitution d'une mémoire organisationnelle ou communautaire? 


\section{Bibliographie}

Auffret G., Bachimont B., « Audiovisual cultural heritage : from tv and radio archiving to hypermedia publishing », In Third European Conference on Research and Advanced Technology for Digital Libraries, vol. 1696 of Lecture Notes in Computer Science, Paris, 1999, p. 58-75.

Bachimont B., «Bibliothèques numériques audiovisuelles: des enjeux scientifiques et techniques », Document Numérique, vol. 2, n 3-4, 1999, p. 219-242.

Baym N. K., «The Emergence of On-Line Community », In. S. G. Jones (ed.), Cybersociety 2.0 : Revisiting Computer-Mediated Communication and Community, Thousand Oaks CA, Sage Publications, 1998, p. 35-68.

Beaudoin V., Velkovska J., «Constitution d'un espace de communication sur Internet», Réseaux, vol. 17, n 97, 1999, p. 121-177.

Berge Z.L., Collins M.P., «Moderating Online Electronic Discussion Group », Paper presented at the American Educational Research Association, Chicago, March 24-28 1997. Document en ligne : http://emoderators.com/moderators/sur_area97.html

Berge Z.L., Collins M.P., «Perceptions of e-moderators about their roles and functions in moderating electronic mailing lists », Distance Education: An International Journal, 21(1), 2000, p. 81-100.

De Luze H., L'ethnométhodologie, Paris, Anthropos, 1997.

De Queiroz J.-M., Ziolkovski M., L’interactionnisme symbolique, Rennes, PUR, 1994.

Donath J., «Identity and Deception in the Virtual Community », In M. A. Smith \& P. Kollock (eds.), Communities in Cyberspace, London - New York, Routledge, 1999, p. 29-59.

DuVal Smith A., "Problems of conflict management in virtual communities », In M. A. Smith \& P. Kollock (eds.), Communities in Cyberspace, London - New York, Routledge, 1999, p. 134-163.

Erickson T., « Social Interaction on the Net : Virtual Community as a Participatory Genre », In. Proceedings of the Thirtieth Hawaii International Conference on System Sciences, 1997, document en ligne : www.pliant.org/personal/Tom_Erickson/VC_as_Genre.html

Erickson T., «Persistent Conversation : an introduction », Journal of Computer-Mediated Communication 4 (4), 1999. Document en ligne : www.ascusc.org/jcmc/vol4/issue4/

Hauch V., Lebraty J.-F., «Présentation de soi et expertise dans les réseaux informatiques », In. N. Guégen \& L. Tobin (eds.), Communication, société et internet, Paris, L'Harmattan, 1998, p. 105-119.

Kerbrat-Orecchioni C., Les interactions verbales, tome 2, Paris, Armand Colin, 1992.

Kollock P., Smith M., « Managing the virtual commons : Cooperation and Conflict in computer communities », In. S. C. Herring (ed.), Computer-Mediated Communication. Linguistic, Social and Cross-Cultural Perspectives, Amsterdam/Philadelphia, John Benjamins, 1996, p. 109-128. 
Lewkowicz M., Zacklad M., «L'écriture collective d'une argumentation dans la prise de decision. Un outil de traçabilité des connaissances en conception », Document Numérique vol. $3, n^{\circ} 3-4,1999$, p. 263-282.

Maingueneau D., L'Analyse de discours. Introduction aux lectures de l'archive, Paris, Hachette, 1991.

Maisonneuve J., La dynamique des groupes, Paris, PUF, 1968.

Marcoccia M., «La Normalisation des comportements communicatifs sur Internet : étude sociopragmatique de la Netiquette», In N. Guégen \& L. Tobin (eds.), Communication, société et internet, Paris, L'Harmattan, 1998, p. 15-32.

Marcoccia M., «La communauté virtuelle: une communauté en paroles », Actes $d u 3^{e}$ Colloque International sur les Usages et Services des Télécommunications - e-usages, Paris 12-14 juin 2001, Paris, France telecom, 2001a, p. 179-189.

Marcoccia M., "L'émergence du rôle d'animateur dans un forum de discussion non modéré », Actes du colloque Coopération, Innovations, Technologies (CITE2001), Troyes, 29-30 novembre 2001, UTT, 2001b, p. 153-170.

Marcoccia M., «La politesse dans les forums de discussion : règles externes, manifestations linguistiques et commentaires métacommunicatifs », In S. Stati, M. Bondi (eds.), Dialogue Analysis 2000 (Proceedings of the IADA Conference, Bologna, June 2000), Tübingen, Niemeyer, 2001c (à paraître).

Marcoccia M., «On-Line Polylogues : Conversation Structure and Participation Framework in Internet Newsgroups », Journal of Pragmatics - special issue on polylogue, 2002 (à paraître).

Morris M., «E-mail editors : gatekeepers or facilitators ?», 1993. Document en ligne : http://emoderators.com/moderators/morris.html

Parret H. et al., Communauté en paroles: communication, consensus, ruptures, Liège, Mardaga, 1995.

Rheingold H., The Art of Hosting Good Conversation Online, 1998. Document en ligne : www.rheingold.com/texts/artonlinehost.html

Smith C.B., McLaughlin M.L., Osborne K.K., «Conduct Control on usenet », Journal of Computer-Mediated Communication vol. 2, n 4, 1997.

Stern Y., «Les quatre dimensions des documents électroniques », Document Numérique vol. $1, \mathrm{n}^{\circ} 1,1997$, p. 55-60.

Vion R., La communication verbale. Analyse des interactions, Paris, Hachette, 1992. 